\title{
Key Technologies of Cache and Computing in 5G Mobile Communication Network
}

\author{
Yanfang Zha 1 \\ School of Artificial Intelligence, Suzhou Industrial Park Institute of Services Outsourcing, Suzhou Jiangsu 215000, China \\ Correspondence should be addressed to Yanfang Zha; zhayf@siso.edu.cn
}

Received 18 August 2021; Revised 10 September 2021; Accepted 15 September 2021; Published 26 September 2021

Academic Editor: Deepak Gupta

Copyright @ 2021 Yanfang Zha. This is an open access article distributed under the Creative Commons Attribution License, which permits unrestricted use, distribution, and reproduction in any medium, provided the original work is properly cited.

\begin{abstract}
The deployment of cache and computing resources in $5 \mathrm{G}$ mobile communication networks is considered as an important way to reduce network transmission delay and redundant content transmission and improve the efficiency of content distribution and network computing processing capacity, which has been widely concerned and recognized by academia and industry. Aiming at the development trend of cache and computing resource allocation in 5G mobile communication networks, in order to improve the efficiency of content cache and reduce network energy consumption, a $5 \mathrm{G}$ network cache optimization strategy based on Stackelberg game was proposed, which modeled network operators and content providers as multimaster and multislave Stackelberg game model. Providers buy base station storage space from network operators to cache popular content. In this paper, we construct the strategy space and profit function of the two sides of the game and prove the existence of Nash equilibrium solution among content providers given a set of base station rental prices of network operators. In this paper, distributed iterative algorithm is used to solve the game model, and the optimal base station pricing of network operators and the optimal base station occupancy rate of content providers are obtained.
\end{abstract}

\section{Introduction}

The network caching strategy reduces the user's request waiting time and content sending waiting time by caching the content of the content provider to the caching node or base station of the $5 \mathrm{G}$ network in advance, reduces the sending energy consumption in the network, and improves the experience quality of network users [1]. The performance of caching strategy directly affects the caching efficiency of network content and the quality of user experience, which is one of the hot spots in the current 5G network research. The traditional content caching strategy is relatively simple to expand in the network, but it has some shortcomings such as low integration, low cache hit rate, and low utilization rate of network resources [2]. At the same time, due to the limited cache resources in the network, there are conflicts among content providers. Previous strategies lack of consideration of the competitive relationship between content providers, there are high cost of network operators, low utilization of cache space, and other drawbacks. For the above problems, some scholars have studied the cache strategies of $5 \mathrm{G}$ networks, but most of these algorithms and strategies only consider a single network performance index and do not consider the competitive relationship between network operators and content providers, and the comprehensive consideration of cache hit rate, network cost, resource utilization, and other factors is insufficient [3]. Therefore, the cache strategy needs further research and optimization.

With the combination of UAV communication and edge caching, coupled with network deployment, resource allocation is very important to the quality of experience (QoE) of content distribution application users [4]. The optimization problem of maximizing QoE is proposed and evaluated by MOS. In the first step, greedy algorithm and Lagrange duality algorithm are used to obtain the optimal position and association. In the second step, the iterative algorithm of optimization is proposed, which achieves the best way of computational complexity and optimization. Network edge caching for D2D can reduce the burden of transmission, which is a more promising technology [5]. But it relies too much on the content preferences of individual users, so we must take some proactive measures if we want to give full 
play to its advantages. In the research, the recommendation algorithm has a great impact on caching decision, so we further study how to improve the caching efficiency of D2D wireless content caching network. According to the requirements of personalized recommendation quality and quantity, including cache capacity, the cache hit rate maximization problem of general network model is adopted to solve the cache efficiency problem. Two criteria in cacheassisted MEC networks are relay link channel gain and direct link channel gain, both of which can greatly improve the computing power [6]. Similarly, there are three criteria for cache-free MEC networks. Based on the outage probability defined by transmission plus computation time, cache can alleviate the impact of heavy computation, while the improvement of cache in high signal-to-noise ratio (SNR) area is not as significant as that of cache-free network adopting the destination with the largest channel gain of direct link. Literature [7] is aimed at the restrictive problem that the development of smart grid technology depends on the convenient data transmission service provided by Power Line Communication (PLC), mainly the channel environment and the increasing traffic load in the backhaul, which is a high energy consumption work. A PLC network with high cache is proposed, which can reduce load energy consumption. Literature [8] is aimed at the increase of network traffic caused by the increase of population, but metropolis adopts local cache to reduce traffic, which is a traditional method and will be limited by storage space. Therefore, researchers put forward data mining technology based on clustering concept. This technology is a pattern of collecting feedback data to reduce traffic, and then, grouping it and redeploying it can achieve the effect of traffic reduction. On the experimental surface, it can indeed reduce the flow rate by about $8 \%$.

As mentioned above, the current cache policy has some problems, such as high cache cost, large network energy consumption, and low cache hit rate. In view of the above problems, this chapter uses the Stackelberg game method to model and analyze the content caching process of $5 \mathrm{G}$ network and comprehensively considers the popularity of content, user preferences, cache hit rate, etc. and puts forward an optimization strategy of $5 \mathrm{G}$ network caching based on Stackelberg game, which is used for network content caching and provides users with high-quality network services.

\section{5G Network Content Caching}

Router nodes and network base stations in 5G networks have storage capacity. By setting the cache strategy and cache replacement strategy, the content of the content provider is cached to the router node or network base station of $5 \mathrm{G}$ network in advance, which can effectively reduce the transmission delay and network energy consumption of 5G network by reducing the retrieval delay of content and the backhaul load of 5G network [9] and at the same time improve the experience quality of netizens.

Cache technology mainly includes three stages: cache policy determination, online user request, and cache replacement. The so-called caching decision refers to making cach- ing strategies based on network traffic changes or content hit rate and caching content into the network in advance. User request is a way to decide the request and send the request content by judging whether the file needed by the user is stored in the cache. Cache replacement refers to replacing cache contents and determining what to update when the cache space reaches a threshold [10].

Caching technology can divide cache into core cache and edge cache according to cache location. Core caching strengthens the overall scheduling ability of the network by caching the network content to the router of the core network, but it enhances the storage load of the core network to a certain extent. Edge caching caches network content to routers close to users' edge networks, which reduces the delay of video search. When the next request is received, the network can respond to users' needs in time, improve the efficiency of users' content acquisition, and increase the transmission speed of network content [11].

Caching technology can be divided into active caching and passive caching according to caching methods. In the case of passive caching, the content within the network is cached in the corresponding location only after being sent through the node. If no data is requested from the responding node, the node does not cache the data. However, due to the high diversity of user preferences, multiple users no longer request exactly the same content, and caching content in passive cache may not meet the needs of future network users [12]. An effective way to improve the efficiency of network caching is to activate the requested content before the user's request arrives. Thus, the active cache can anticipate user requirements and fetch content from the content provider before being requested.

User demand and user mobility are two factors to consider activating and caching network users in 5G networks.

The advantages of applying caching technology in 5G networks are as follows:

(1) It can reduce the demand of nodes for backhaul links, so it can increase the deployment density of nodes and improve spectrum utilization and network throughput

(2) Hot data can be cached in advance in the cache node to balance the traffic in the network, thus avoiding the occurrence of network congestion during the peak traffic period

(3) It can reduce the network occupation of backhaul bandwidth and reduce the deployment cost of backhaul link of base station to a certain extent

(4) It can effectively reduce the retrieval delay and transmission delay for users to obtain content, because users can directly obtain the requested content from the cache node and do not need to obtain it from the core network

(5) It can effectively avoid duplicate contents transmitted on the backhaul link, thereby reducing the energy consumption of the network and improving the energy efficiency 


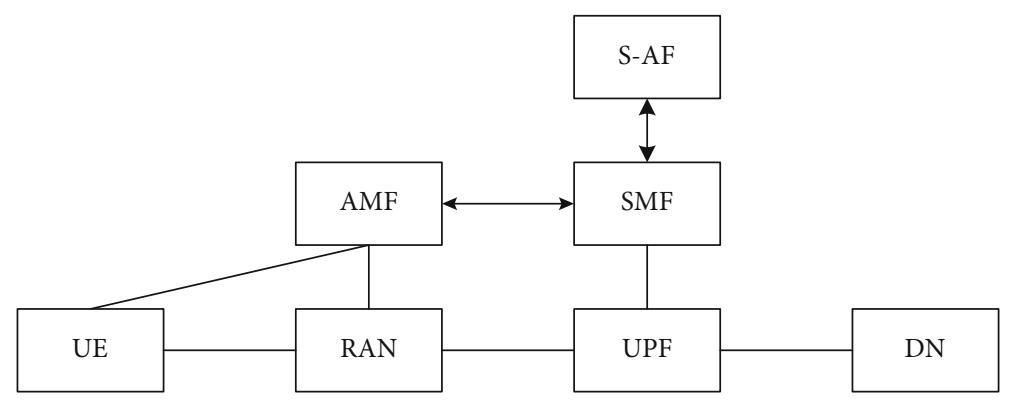

FIgURE 1: 5G network architecture.

\section{System Model of Content Caching}

The focus of this paper is to model and analyze the content caching system. Firstly, the network architecture of content caching in $5 \mathrm{G}$ network is summarized, and then, the system is modeled in this network architecture. Finally, the popularity of content, user preference, and cache hit rate are introduced in detail.

As shown in Figure 1, the 5G network architecture [13] mainly includes modules such as UE, AMF, RAN, S-AF, SMF, UPF, and DN. The functions of each module are as follows.

(1) UE. The module mainly includes $5 \mathrm{G}$ user terminals requesting network content.

(2) AMF. AMF module is mainly responsible for user mobility management and network access control. The $5 \mathrm{G}$ core access and mobility management function (AMF) receives all connection and sessionrelated information from user equipment, but is only responsible for handling connection and mobility management tasks. All messages related to session management are forwarded to the session management function through the reference interface.

(3) RAN. It is mainly responsible for the terminal access function of $5 \mathrm{G}$ network, and the base stations of network operators are distributed in the layer of wireless access network.

(4) Stackelberg Game Application [14] Function. S-AF module mainly interacts with the implementation of cache optimization strategy, the processing of network information, and the core network of $5 \mathrm{G}$ network presented in this section to provide corresponding services.

(5) SMF. This module is mainly responsible for the management and control of end-user sessions.

(6) UPF. The user interface function completes the related operations of the user interface, including the retrieval and root functions of the data content of the user interface.

(7) DN. The content provider is located in this location, mainly providing the content required by users.
3.1. System Model. Network operators in wireless access networks and content providers in data networks are combined as a system model. Figure 2 shows the system model of cache optimization strategy.

The system model in this paper includes $M$ network operators $\{1,2, \cdots, M\}, N$ content providers $\{1,2, \cdots, N\}$, and 5G network [15] end users. Each network operator may lend base station space to the content provider to precache content specified by the content provider. Here, the base station distribution of network operators follows the distribution density. The different $\lambda$ is arranged neatly, and the users follow the uniform distribution of berthing points with different properties of distribution density $n$.

In the system model, the specific process for completing content request and download is the first step: the network operator first sets the price of its base station and notifies the content provider through the $5 \mathrm{G}$ network. Second step: The content provider leases a certain percentage of the base stations based on the type of network operator and the pricing of the base stations and caches popular or popular content to the base stations for request by the end user. Step 3: The end user downloads the required content from the base station.

3.2. Content Popularity. In practical applications, users have different preferences for different contents (for example, some people like football videos, scientific messages, or music), and part of the contents that the next user may request is cached and provided to the user based on the user's historical browsing records, thus reducing the request waiting time and transmission waiting time of the content [16]. The contents of popular files on the network are set to $C$, the set represents $C=\left\{F_{1}, F_{2}, F_{c}\right\}$, and the popularity of the $C$ content is set to $D_{c}, c=\{1,2, \cdots, C\}$.

$$
D_{c}=\frac{1 / c^{\alpha}}{\sum_{j=1}^{C}\left(1 / j^{\alpha}\right)}, \forall c .
$$

As can be seen from Equation (1), content $F_{1}$ is the most popular and content $F_{c}$ is the least popular.

In this paper, the adjustment coefficient is introduced based on Formula (1). For $\sigma$, in order to adjust the cached content, the base station caches the popular content of the content provider and improves the income of the content 


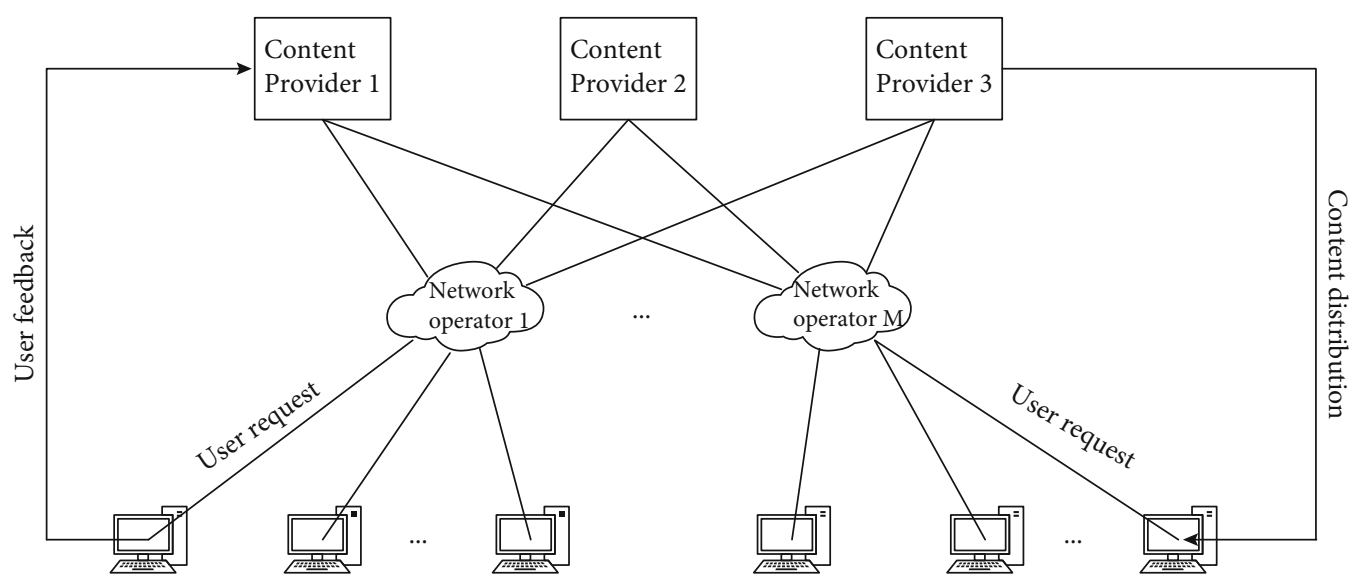

Figure 2: System model.

provider. The popularity of imported $\sigma$ modified content is expressed in Formula (2).

$$
D_{c}=\frac{\sigma / c^{\alpha}}{\sum_{j=1}^{C}\left(1 / j^{\alpha}\right)}, \forall c .
$$

3.3. User Preference Degree. According to users' usage habits, users have different preferences for content providers. For example, for video providers, Tencent, Youku, and eye contact use groups are different. User preference indicates the user's experience level with the content provider, and the higher the preference, the higher the user's access frequency. Therefore, in this specification, by introducing a user preference indicator, content provided by a content provider with a high user preference degree is cached in advance in a network in order to improve the revenue of the content provider. Assuming there are $N$ content providers $n=\{1$, $2, \cdots, N\}$, the user preferences are $e=\left\{E_{1}, E_{2} \cdots E_{N}\right\}$, respectively. Since the user's content provider preferences follow the Zipf distribution, $E_{n}$ is defined as Formula (3).

$$
E_{n}=\frac{1 / n^{\beta}}{\sum_{i=1}^{n}\left(1 / i^{\beta}\right)}, \forall n
$$

Among them, $\beta$ benchmark index, $\beta>0$. The larger the beta, the more popular the content provider is among users and the more frequently it is accessed. According to Formula (3), it can be seen that content provider 1 is the most popular and content provider $N$ is the least popular.

The cache hit ratio of content providers is an indicator that content caching must consider. The cache hit ratio $P_{\text {hit }}$ is shown in Formula (4).

$$
P_{\text {hit }}=\frac{\pi \lambda P_{c} R_{\mathrm{th}}^{2} \tau e^{-\lambda \pi P_{c} R_{\mathrm{th}}^{2}}}{\varepsilon \tau+\mu},
$$

where $\mu$ represents the normalization factor and $e^{-\lambda \pi P_{c} R_{\mathrm{th}}^{2}}$ is the adjustment factor for the cache hit ratio.

\section{Creation of Stackelberg Game}

The standard game model should include three elements: game participants, game strategy space, and game income function. Among them, Stackelberg is a game suitable for master-slave relationship $[17,18]$. The participants of the game are generally instructors and followers, and the instructors and followers have their own game strategies and profit functions. The process of the game is as follows:

(1) Leaders make decisions based on their own benefits

(2) Followers make corresponding decisions according to leaders' decision-making actions

(3) Maximize the income value of both parties through the game process

4.1. Composition of Stackelberg Game. In this paper, the game model is established as a multimaster and multislave Stackelberg game $[19,20]$, and the constituent elements of the game are as follows:

(1) Participants in the Game. The leaders are $N$ content providers $\{1,2, \cdots, N\}$. Followers are $M$ network operators $\{1,2, \cdots, M\}$.

(2) Strategy Space of Game. The leader's strategy is the proportion of content provider renting network operator's base station $t_{m n}$, or the follower's strategy is to set the rental price of network operator's base station, which is expressed as $P=\left\{P 1, P 2, \cdots \cdots P_{M}\right\}$.

(3) The Return Function of the Player. Revenue from caching content

$$
U_{n}^{\text {cache }}=\sum_{m=1}^{M} \sum_{c=1}^{C} K \eta Q E_{n} D_{c} P_{\text {hit }}
$$

where $Q$ is the unit price of each content and $K$ is the average number of users' requests for content. The 
other part is the rent for renting the base station of the network operator:

$$
U_{n}^{\mathrm{rent}}=\sum_{m=1}^{M} \tau_{m n} \lambda_{m} P_{m} .
$$
follows:

The revenue function for content providers is as

$$
U_{n}=U_{n}^{\text {cache }}-U_{n}^{\text {rent }}
$$

For network operators, the revenue mainly comes from the rental fee $P_{m}$ charged by the content provider for renting the base station and the cost $C_{m}$ of managing the base station by the network operators. Therefore, the revenue function of network operators is as follows:

$$
U_{m}=\sum_{n=1}^{N}\left(P_{m}-C_{m}\right) \tau_{m n} \lambda_{m} .
$$

4.2. Problem Creation of Stackelberg Game. For a single network operator, the optimization problem can be created as a revenue maximization problem for the network operator, namely,

$$
\max _{p \geq 0} U_{m}(p, \tau)
$$

Special attention should be paid to the fact that the content storage capacity must not exceed the total cache space of the base station.

For each content provider, the optimization problem is created as the revenue maximization problem of the content provider. By substituting Formulas (5) and (6) into Formula (7) and deducing calculation, the revenue of the content provider can be obtained as follows:

$$
\begin{aligned}
U_{n} & =\sum_{m=1}^{M} \sum_{c=1}^{C} K \eta Q E_{n} D_{c} P_{\mathrm{hit}}-\sum_{m=1}^{M} \tau_{n n} \lambda_{m} P_{m} \\
& =\sum_{m=1}^{M}\left[\frac{\sum_{c=1}^{C} K \eta Q E_{n} D_{c} \pi \lambda_{m} P_{c} R_{h}^{2} \tau e^{-\lambda \pi P_{c} R_{h h}^{2}}}{\varepsilon \tau+\mu}-\tau_{m n} \lambda_{m} P_{m}\right]
\end{aligned}
$$

The optimization problem for the content provider is created as follows:

$$
\max U_{n}(p, \tau)
$$

Among them, $0 \leq 1$.

According to the above game model, the goal of the network operator is to maximize the benefits specified in Formula (8). The content provider leases the network operator's base station in a proportion $\tau$ depending on the network operator's leased price $P_{m}$ of the base station. The purpose of the game is to find Stackelberg's Nash equilibrium point, discourage leaders and followers from deviating from the best strategy, and achieve the goal of maximizing the interests of both parties.

4.3. Stackelberg's Nash Equilibrium. The purpose of the game $[21,22]$ is to let the participants choose the best strategy. Whether the game ends or not determines whether the game process has a stable state. It is worth studying to play games only at the end of the game process. The steady state in game is usually described by Nash equilibrium.

Nash equilibrium refers to a combination of strategies called Nash equilibrium, which can make the strategies of all players correspond to those of other players. Once Nash equilibrium is reached, the strategy chosen by any participant is the most appropriate, and it is impossible to obtain higher benefits by adjusting his own strategy. Nash's equilibrium strategy is divided into "pure strategic Nash equilibrium" and "mixed strategic Nash equilibrium." Mixed strategy means that game participants choose response strategies randomly through probability distribution, while pure strategy means that game participants choose a single strategy determined in the current strategy space.

It is worth noting that before solving any nanoequilibrium, it is necessary to prove whether nanoequilibrium exists in the game process. For noncooperative games, Nash equilibrium is defined as no operating point, and participants can change their strategies to increase returns.

For noncooperative games, in general, if the game satisfies the following conditions,

(1) The set of players in the game is limited

(2) The strategy space set of game belongs to the bounded closed set of Euclidean space

(3) The profit function of noncooperative game is continuous in strategy space and satisfies the characteristics of concave function

It can be concluded that there is Nash equilibrium in the game process, and the utility of each player will be maximized, and any participant cannot obtain higher returns by changing his own strategy privately.

If there are Nash equilibrium solutions in both game processes, then there must be Nash equilibrium solutions in their combined game.

The Nash equilibrium of Stackelberg game is obtained by looking for the perfect Nash equilibrium. In the game set here, after the lease price of the base station of the network operator set is given, a noncooperative game relationship is provided between the content providers. However, in a practical case, a plurality of nanoequilibrium solutions may exist even if there is not necessarily a nanoequilibrium solution among content providers. Therefore, this paper analyzes and proves the existence of Nash's equilibrium solution.

The return function satisfies the strict concave function characteristic, and the return function $U_{n}$ is calculated as 
the first derivative and the second deflection derivative of the strategy space.

$$
\begin{aligned}
& \frac{d U_{n}}{d \tau_{m n}}=\sum_{m=1}^{M}\left[\frac{\mu \sum_{c=1}^{C} K \eta Q E_{n} D_{c} \pi \lambda P_{c} R_{\mathrm{th}}^{2} e^{-\lambda \pi P_{c} R_{\mathrm{th}}^{2}}}{(\varepsilon \tau+\mu)^{3}}\right]-\lambda_{m} P_{m}, \\
& \frac{d U_{n}}{d \tau^{2}}=\sum_{m=1}^{M}\left[\frac{-2 \mu \sum_{c=1}^{C} \pi K \eta Q E_{n} D_{c} \lambda P_{c} R_{\mathrm{th}}^{2} e^{-\lambda \pi P_{c} R_{\mathrm{th}}^{2}}}{(\varepsilon \tau+\mu)^{3}}\right]<0 .
\end{aligned}
$$

Through solving, it is found that the result of quadratic partial differential is negative. The income function $U_{n}$ satisfies strict sag function characteristics. Therefore, the game between content providers has Nash equilibrium, which ensures the existence of perfect Nash equilibrium in the Stackelberg game model. The Nash equilibrium proposed in this paper is mainly aimed at the balance between content providers, and it is also the main function of the Stackelberg game model.

\section{Optimization of Stackelberg Game}

In the process of solving game optimization, it is necessary to consider the actual situation, such as the space size of the base station, the high price, and the low adjustment. First, the maximum and minimum values of the rental price are determined by determining the pilot frequency without considering the limitation of the storage space size of the base station. After that, the theory is expanded and the base station space is limited. Considering the actual situation of the lease ratio, it is solved in detail by the Lagrange multiplier method. Finally, the best rental ratio and the best pricing of the base station are obtained. The process of solving the game is as follows.

It is assumed that the limitation of the storage size $S$ of the network operator is not considered. Firstly, the network operator gives a set of base station renting prices $P=\left\{P_{1}\right.$, $\left.P_{2}, \cdots, P_{M}\right\}$, and then, by deriving $d U_{n} / d \tau_{m n}=0$, the optimal solution $\tau$ of the content provider renting base station ratio is solved:

$$
\tau=\frac{1}{\varepsilon} \sqrt{\frac{\mu K \eta \pi Q E_{n} \lambda P_{c} R_{\mathrm{th}}^{2} e^{-\lambda \pi P_{c} R_{L}^{2}} \sum_{c=1}^{C} D_{c}}{\lambda_{m} P_{m}}-\mu .}
$$

When $\tau=0$, because the price of the base station is too high, the content provider chooses which base station not to rent to store the content, and because of the large delay of content request and search, the experience quality of the user is reduced. Therefore, there is a maximum rental price of the base station. Substituting $\tau=0$ into Equation (14), the maximum rental price is as follows:

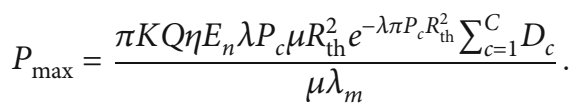

When $\tau=0$ in one case, the content provider chooses to lease all the base stations to store the content because the price of the base stations is too low, thus reducing the revenue of the network operator. Therefore, the base station price has a minimum value. Substituting $\tau=1$ into Equation (14), the minimum value of the rental price is as follows:

$$
P_{\min }=\frac{\pi K Q \eta E_{n} \lambda P_{c} \mu R_{\mathrm{th}}^{2} e^{-\lambda \pi P_{c} R_{\mathrm{th}}^{2}} \sum_{c=1}^{C} D_{c}}{\lambda_{m}(\mu+\varepsilon)^{2}} .
$$

By the above solver process, the maximum and minimum values of the rental price of the base station are determined. If the price of the base station is lower than $P_{\min }$, the network operator should increase the price of the current base station. If the base station price is higher than $P_{\max }$, the network operator should reduce the current base station price. Through multiple games to modify the price strategy, the network operators and content providers ultimately maximize the benefits of both sides.

The above situation is only theoretical analysis. In practical application, the cache space of base station is limited. Therefore, the storage space size $S_{m}$ is limited, and the specific constraints are as follows:

$$
S_{m}=\sum_{n=1}^{N} \tau_{m n} \lambda_{m} \leq S_{\max }
$$

where $S_{\max }$ is the maximum storage space.

In the actual solver, considering the limitation of storage space, the lease ratio of base station must meet $0<T \leq 1$. Therefore, this paper seeks a solution by choosing the Lagrange multiplier method. First, a Lagrangian function of the formula is created, that is, Formula (17).

$$
\begin{aligned}
L_{v}= & \sum_{m=1}^{M} \sum_{c=1}^{c} K q \eta E_{n} D_{c} P_{\text {hit }}-\sum_{m=1}^{M} \tau_{m n} \lambda_{m} P_{m} \\
& +A \tau_{m n}+B \tau_{m n}+\theta \tau_{m n} \lambda_{m}
\end{aligned}
$$

where $A, B$, and $\theta$ are Lagrange multipliers. According to the constraint conditions, the sufficient and necessary KKT (Karush-Kuhn-Tucher) condition of this function is as follows:

$$
\left\{\begin{array}{l}
\frac{d L_{U}}{d \tau}=0, \\
A, B \geq 0, \\
A \tau=0, \\
B(\tau-1)=0 .
\end{array}\right.
$$


The optimum value $\tau^{*}$ of the base station lease ratio is derived from the KKT condition, as shown in Formula (19).

$$
\tau^{*}=\left\{\begin{array}{l}
0, \theta>\frac{K Q \pi \lambda \eta E_{n} P_{c} R_{\mathrm{th}}^{2} e^{-\lambda \pi P_{c} R_{\mathrm{th}}^{2}} \sum_{c=1}^{C} D_{c}}{\varepsilon \lambda_{m} P_{m}}-1, \\
1, \theta<\frac{\varepsilon K Q \pi \lambda \eta E_{n} P_{c} R_{\mathrm{th}}^{2} e^{-\lambda \pi P_{c} R_{\mathrm{th}}^{2}} \sum_{c=1}^{C} D_{c}}{(\varepsilon+\mu)^{2}+\lambda_{m} P_{m}}-1, \\
\frac{1}{\mu}\left(\sqrt{\frac{\varepsilon K Q \pi \lambda \eta E_{n} P_{c} R_{\mathrm{th}}^{2} e^{-\lambda \pi P_{c} R_{\mathrm{th}}^{2}} \sum_{c=1}^{C} D_{c}}{1+\theta}}-\varepsilon\right), \text { other. }
\end{array}\right.
$$

The variable $\theta$ is a constraint condition set to satisfy the space limitation of the base station, as shown in Formula (20).

$$
\theta=\left(\frac{1}{\mu\left(S-\sum_{m=1}^{M} \lambda_{m} P_{m}\right)}\right)^{2}-1 .
$$

After solving the optimal value $\tau^{*}$, the best solution of base station price must be determined. The maximum value is obtained from Equation (20), the conductance result is shown in Equation (21), the derivative of Equation (8) is set to 0 , the inverse solution is $P_{m}$, and the best price of the base station is set to $P_{m}$ (Formula (22)).

$$
\begin{aligned}
\frac{d U_{m}}{d P_{m}} & =\tau_{m n}^{*} \lambda_{m}+\lambda_{m}\left(P_{m}-C_{m}\right) \frac{d \tau^{*}}{d P_{m}}, \\
P_{m}^{*} & =C_{m}-\frac{\tau^{*}}{d \tau^{*} / d P_{m}} .
\end{aligned}
$$

The above solution shows that the best solution of base station price does not have a closed form, because the best price of a single network operator's base station is closely related to the prices of other base stations. That is, after the network operator's price changes, other network operators need to update their base station rental prices. Therefore, the price of network operators needs to be solved iteratively. The repetition is as follows:

$$
P_{m}^{t+1}=C_{m}^{t}-\frac{\tau^{*}\left(P_{m}^{t}\right)}{d \tau^{*} / d P_{m}^{t}}
$$

where $t$ represents the number of iterations, $C_{m}^{t}$ represents the cost price of the base station managed by the network operator during the $t$ iteration, and $P_{m}^{t}$ represents the rental price of the base station during the $T$ iteration.

If the revenue of the network operator and content provider is maximized at the time point $T+1$, the repetitive process is stopped. Otherwise, the next iteration period will proceed until the two benefits reach the maximum value. Stop repeating the process.

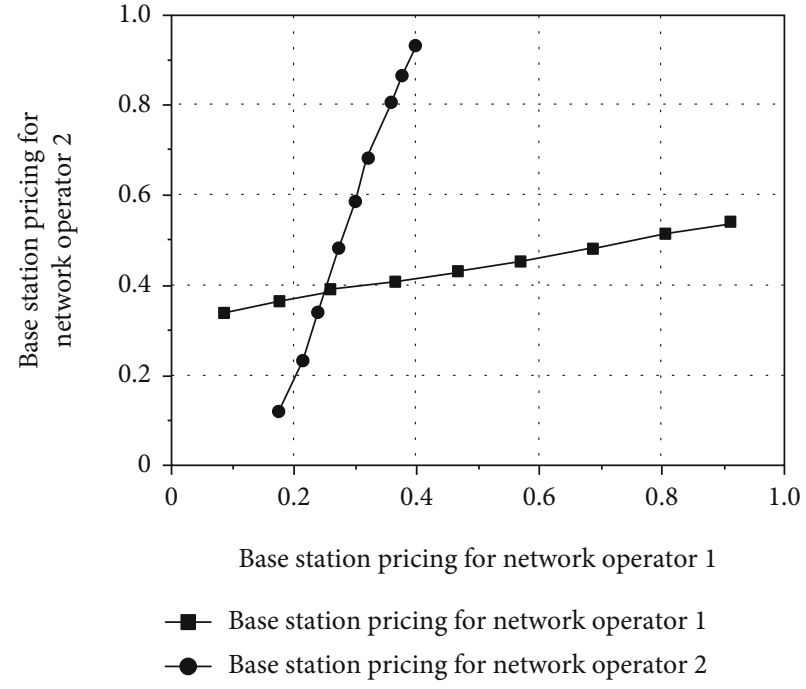

Figure 3: Relationship between optimal base station pricing of network operators.

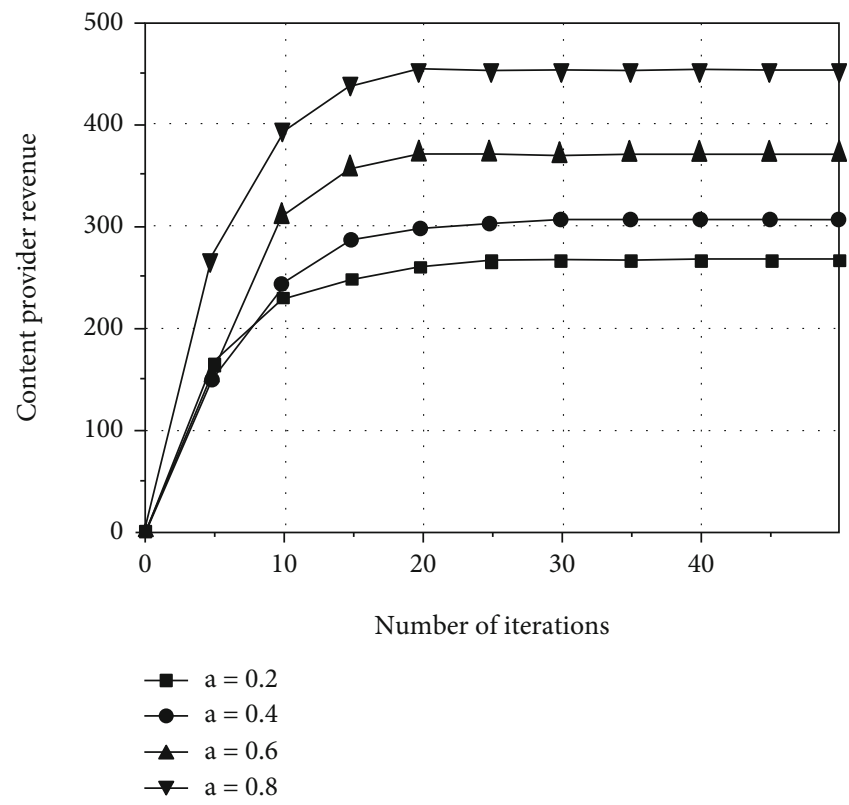

FIgURE 4: Relationship between iteration times and content provider revenue.

\section{Analysis of Simulation Results}

In this paper, we use the simulation tool MATLAB to verify and analyze the proposed algorithm. The simulation of this paper is a network environment composed of two network operators and three content providers. The content provider conflicts with the cache space resources of the network operator to cache the content. The setting values of the simulation parameters set the initial cache requirement policy for each network operator to 1.2 GB, where the available cache space for two network operators is 500 GB. $\alpha=2.3, \beta=0.7$, $C=3,6=15, \mu=8, R_{n}=10, P_{c}=0.8, \lambda=8, \eta=20$, and $K=50$. The income value of this paper is dimensionless, 


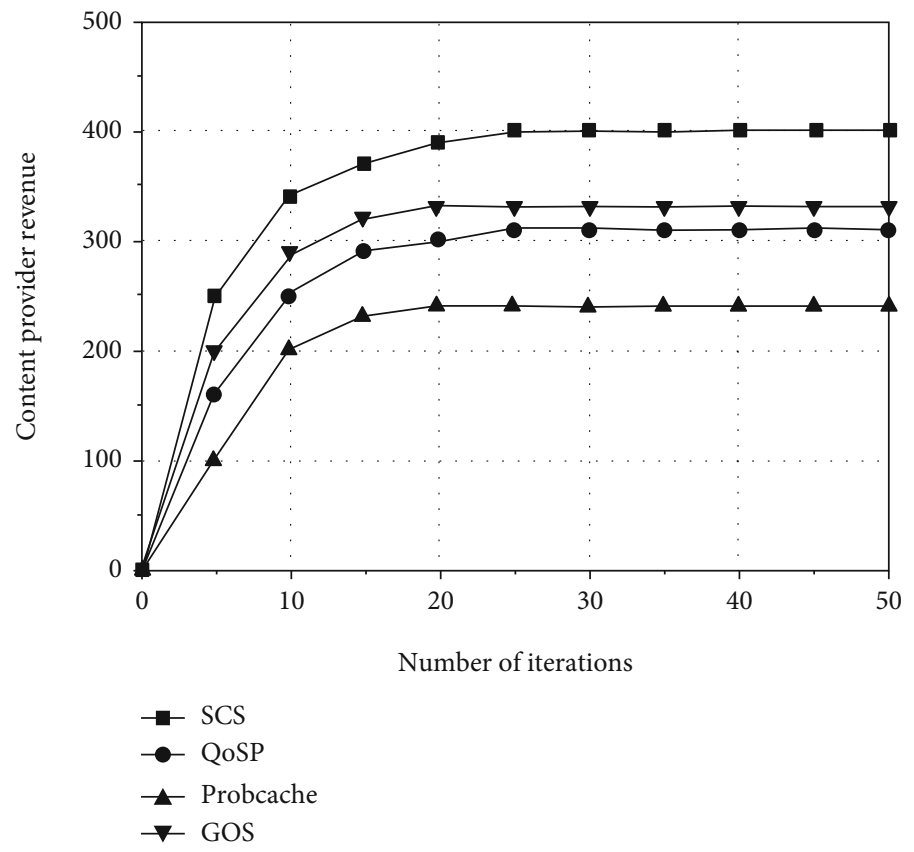

Figure 5: Relationship between iteration times and content provider revenue.

and the larger the value, the higher the corresponding income. In the simulation, the algorithm (SCS) compares the user QoS priority algorithm (QOSP) [23] and ProbCache $(P=0.38)$ with the Global Optimization Scheme (GOS) [24] and compares the average energy consumption reduction rate, network cost, and average cache hit rate, where the average cache hit rate and the average energy consumption reduction rate are defined as follows:

(1) Average Cache Hit Ratio $P_{\text {hit }}$. It represents the average hit probability of users requesting cached content in the network and is used to measure the rationality of content caching strategy in the network. The cache hit ratio is defined as follows:

$$
P_{\text {hit }}=\frac{\sum_{B} \text { hit }_{c, i}^{\text {count }}}{\sum_{B} \text { request }_{\text {sum }, i}^{\text {count }}} \text {, }
$$

where $\sum_{B}$ hit $_{c, i}^{\text {count }}$ denotes the total number of times the user requests the content file from the cache base station $i$ and request $\mathrm{sum}, i^{\text {count }}$ denotes the total number of times the user successfully obtains the content from the cache base station $i$.

(2) Average Energy Consumption Reduction Rate $E_{\text {ave }}$. It represents the change of energy consumption between simulation comparison algorithm and classical LCE, as shown in Formula (25).

$$
E_{\mathrm{ave}}=\frac{E_{\mathrm{LCE}}-E_{\mathrm{other}}}{E_{\mathrm{LCE}}} .
$$

The energy consumption of the algorithm is composed of the cache energy consumption $E_{\text {cache }}$ and the transmission energy consumption $E_{\text {tran }}, f_{n}$ represents the number of cached files, and $e_{\text {cache }}$ represents the energy consumption required for caching each file. Energy consumption is defined as follows:

$$
E_{\text {other }}=E_{\text {tran }}+E_{\text {cache }}=E_{\text {tran }}+f_{n} e_{\text {cache }} \text {. }
$$

Figure 3 is a diagram illustrating the relationship between the best base station pricing of the network operator 1 and the best base station pricing of the network operator 2. The points on the two curves in the graph, respectively, represent the best pricing strategy of the network operator to other network operators. It can be seen from the figure that the two curves have an intersection $(0.28,0.36)$, which is the Nash equilibrium point of the game, where the benefits of both can be maximized. Once the price of the network operator 2 is determined, the size of the revenue value of the network operator 1 is affected by the change of base station pricing, and the revenue itself is maximized according to the optimal pricing of the base station. If the price of the network operator 2 is changed, although the optimal base station pricing of the network operator 1 is changed, there is an optimal pricing that maximizes its revenue.

The intersection point in Figure 3 is $(0.28,0.36)$, and the intersection point is the Nash equilibrium point of the game, where the benefits of both can be maximized.

Figure 4 is a diagram showing popularity indices of different contents: the relationship between the number of repetitions and the revenue of the content provider in the case of $\alpha$. As can be seen from Figure 4, with the increase of repetition times, the rental ratio of content providers and the base station pricing of network operators gradually repeat to the optimal value. Therefore, the revenue value of 


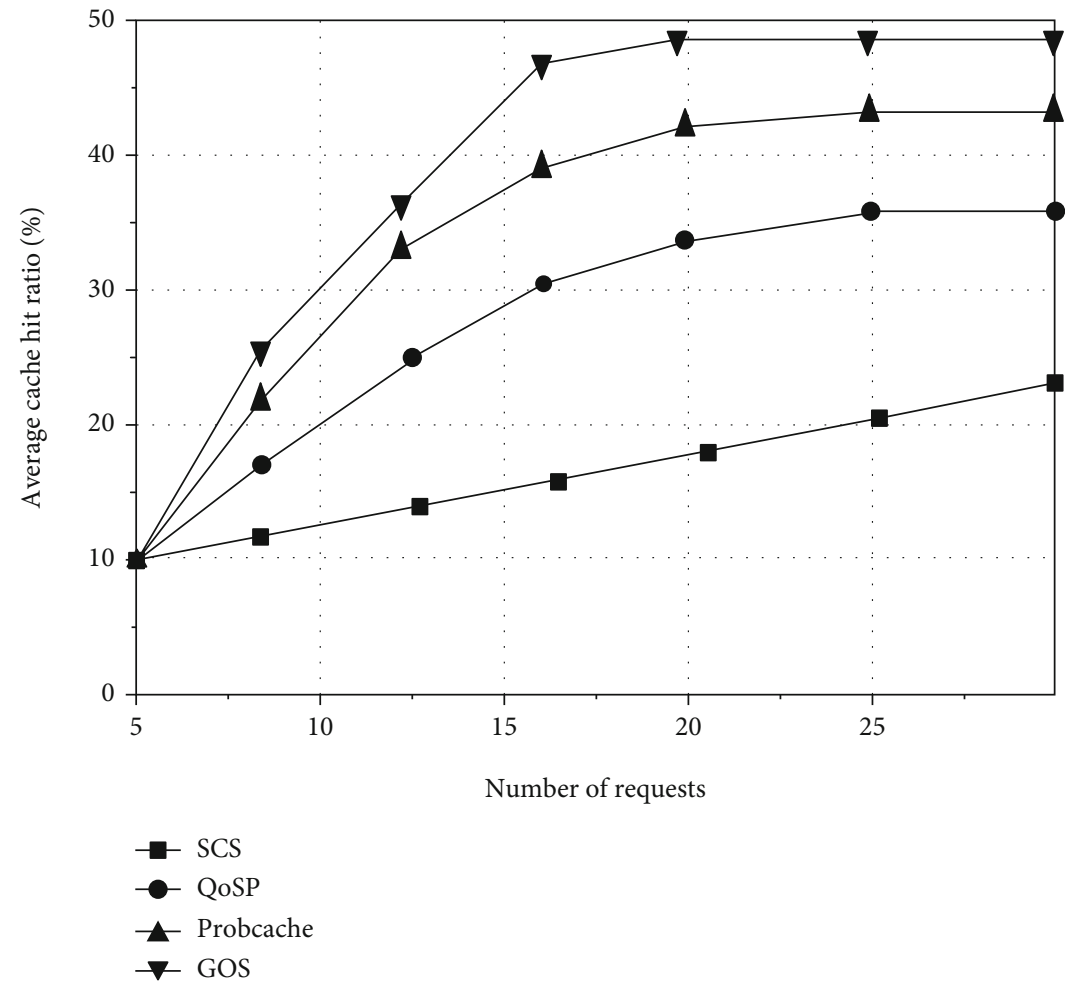

Figure 6: Number of requests vs. average cache hit ratio.

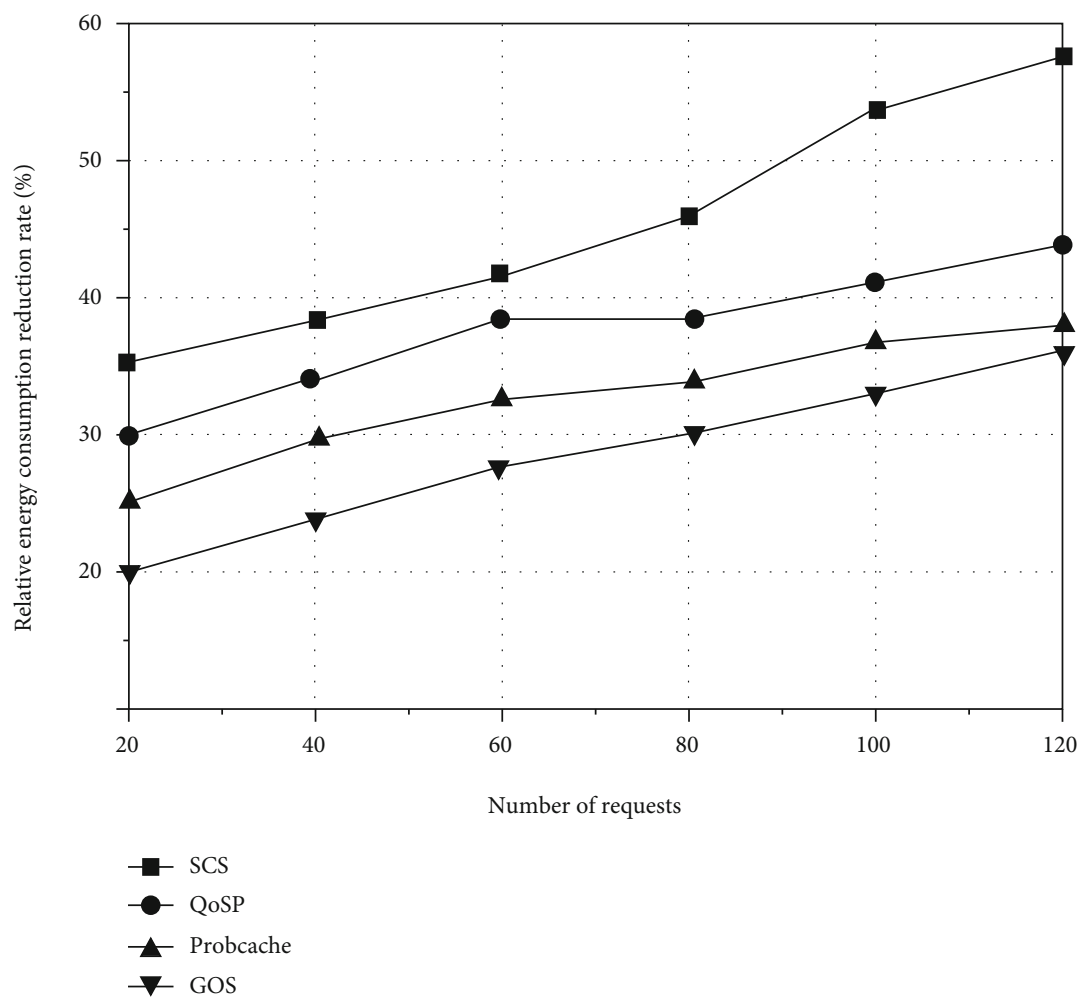

FIgURE 7: Relationship between content request times and relative energy consumption reduction rate.

the content provider continues to increase, and when the number of repetitions reaches about 24 times, the revenue function value of the content provider converges to the optimal value. Displaying the $\alpha$ value in the figure will also directly affect the revenue of the content provider. The larger the $\alpha$ value, the more popular the content provided by the 
content provider, and thus the higher the revenue value of the content provider within the network.

Figure 5 is a diagram illustrating the number of repetitions of different algorithms and alpha's revenue relationship with the content provider. As can be seen from Figure 5, in the case of different algorithms, the ProbCache policy only determines the content cached by the network based on the weighted probability value of the content and does not fully consider the conflict relationship between the content providers in the network. QoSP policy caches content according to users' QoS requirements, which improves the revenue of content providers to a certain extent. However, GOS policy fully considers the competitive relationship among content providers in the network, and the revenue of content providers is higher than QoSP policy. Because the GOS strategy has some limitations in taking global revenue as the objective function of the strategy, the revenue value of content providers is lower than that of this strategy.

Figure 6 is a diagram illustrating the relationship between the number of content requests and the average cache hit rate in different algorithms. As shown in Figure 6, the number of requests increases and the cache hit ratio increases gradually. ProbCache determines the content of cache according to the weighted probability value, which effectively improves the cache hit rate of content, so the cache hit rate is the highest. The lowest hit rate of GOS cash is because GOS strategy only considers the overall income and does not consider the index of cash hit rate in detail. QoSP considers content hit ratio, but because of the request for latency value in user QoS, it can sacrifice a part of the revenue of content provider to meet the latency limit. The strategy of this paper fully considers the cash hit rate. The game model introduces the popularity and preference of content into the income function and effectively improves the cache hit rate of the network by influencing the income of content providers through the feedback of cash rate index.

Figure 7 shows a small relationship between the number of content requests and the relative energy consumption in different algorithms. As can be seen from Figure 7, with the increase of requests, the reduction rate of relative energy consumption also increases. Compared with other schemes, the reduction rate of energy consumption of the algorithm adopted in this paper has obvious advantages. Because other strategies do not consider the index of energy consumption, the energy consumption performance is relatively poor.

\section{Conclusion}

According to the development trend of caching and computing resource allocation in $5 \mathrm{G}$ mobile communication networks, in order to improve the efficiency of content caching and reduce network energy consumption, this paper proposes a strategy of optimizing $5 \mathrm{G}$ network caching based on Stackelberg game, which models network operators and content providers as the multimaster and multislave Stackelberg game model. Providers purchase base station storage space from network operators to cache popular content, so as to achieve high-performance computing of $5 \mathrm{G}$ communi- cation network and optimize resources, and the reduction rate of energy consumption has obvious advantages compared with other schemes.

\section{Data Availability}

The data used to support the findings of this study are available from the corresponding author upon request.

\section{Conflicts of Interest}

The author declares no conflicts of interest.

\section{References}

[1] R. Torre, I. Leyva-Mayorga, S. Pandi, H. Salah, G. T. Nguyen, and F. H. P. Fitzek, "Implementation of network-coded cooperation for energy efficient content distribution in $5 \mathrm{G}$ mobile small cells," IEEE Access, vol. 8, pp. 185964-185980, 2020.

[2] Q. Zhou, L. Chen, G. Li, and Z. Zhang, "Research on Internet of Things technology," in Computer, Informatics, Cybernetics and Applications. Lecture Notes in Electrical Engineering, X. He, E. Hua, Y. Lin, and X. Liu, Eds., vol. 107, pp. 253-260, Springer, Dordrecht, 2012.

[3] S. Maharjan, Y. Zhang, and S. Gjessing, "Economic approaches for cognitive radio networks: a survey," Wireless Personal Communications, vol. 57, no. 1, pp. 33-51, 2011.

[4] T. Zhang, Y. Wang, Y. Liu, W. Xu, and A. Nallanathan, "Cache-enabling UAV communications: network deployment and resource allocation," IEEE Transactions on Wireless Communications, vol. 19, no. 11, pp. 7470-7483, 2020.

[5] Y. Fu, L. Salaun, X. Yang, W. Wen, and T. Q. S. Quek, "Caching efficiency maximization for device-to-device communication networks: a recommend to cache approach," IEEE Transactions on Wireless Communications, p. 1, 2021.

[6] J. Xia, C. Li, X. Lai et al., "Cache-aided mobile edge computing for B5G wireless communication networks," EURASIP Journal on Wireless Communications and Networking, vol. 2020, no. 1, 2020.

[7] Y. Qian, L. Shi, L. Shi, K. Cai, J. Li, and F. Shu, "Cache-enabled power line communication networks: caching node selection and backhaul energy optimization," IEEE Transactions on Green Communications and Networking, vol. 4, no. 2, pp. 606-615, 2020.

[8] L. Kang, R. S. Chen, Y. C. Chen, C. C. Wang, X. Li, and T. Y. $\mathrm{Wu}$, "Using cache optimization method to reduce network traffic in communication systems based on cloud computing," IEEE Access, vol. 7, pp. 124397-124409, 2019.

[9] I. Shayea, M. Ergen, M. Hadri Azmi, S. Aldirmaz Colak, R. Nordin, and Y. I. Daradkeh, "Key challenges, drivers and solutions for mobility management in $5 \mathrm{G}$ networks: a survey," IEEE Access, vol. 8, pp. 172534-172552, 2020.

[10] F. H. Sun, "Research on key technologies of optimal routing design in ad hoc networks," Applied Mechanics \& Materials, vol. 380-384, pp. 2312-2315, 2013.

[11] M. Lou and J. Xiao, "Dynamic, tagless cache coherence architecture in chip multiprocessor," Advances in Intelligent Systems and Computing, vol. 277, pp. 201-209, 2014.

[12] N. Li, Y. Wang, S. Li, and Z. Wang, "Research on relations between Two PWM technologies and system performance optimization," in Proceedings of the 7th International Power 
Electronics and Motion Control Conference, pp. 2333-2337, Harbin, China, 2012.

[13] A. Gupta and R. K. Jha, "A survey of 5G network: architecture and emerging technologies," IEEE Access, vol. 3, pp. 12061232, 2015.

[14] H. Sedghani, D. Ardagna, M. Passacantando, M. Z. Lighvan, and H. S. Aghdasi, "An incentive mechanism based on a Stackelberg game for mobile crowdsensing systems with budget constraint," Ad Hoc Networks, vol. 123, article 102626, 2021.

[15] M. Luo, Y. Pei, and W. Huang, "Mutual heterogeneous signcryption schemes with different system parameters for $5 \mathrm{G}$ network slicings," Wireless Netw, vol. 27, no. 3, pp. 1901-1912, 2021.

[16] X. Wang, P. Sun, and Y. Zhang, "Notice of retraction: research on key technologies of polymorphous and heterogeneous data collection for ocean ship," in International conference on logistics Engineering and intelligent transportation systems, pp. 1-5, Wuhan, China, 2010.

[17] X. Kang, R. Zhang, and M. Motani, "Price-based resource allocation for spectrum-sharing femtocell networks: a Stackelberg game approach," IEEE Journal on Selected Areas in Communications, vol. 30, no. 3, pp. 538-549, 2012.

[18] M. He, Y. Jin, H. Zeng, and J. Cao, "Pricing decisions about waste recycling from the perspective of industrial symbiosis in an industrial park: a game model and its application," Journal of Cleaner Production, vol. 251, article 119417, 2020.

[19] L. Xia and L. Hai, "A seamless transfer strategy based on multimaster and multi-slave microgrid," in 2018 9th IEEE International Symposium on Power Electronics for Distributed Generation Systems (PEDG), pp. 1-5, Charlotte, NC, USA, 2018.

[20] J. Ma and H. Ren, "The impact of variable cost on a dynamic Cournot-Stackelberg game with two decision-making stages," Communications in Nonlinear Science and Numerical Simulation, vol. 62, pp. 184-201, 2018.

[21] S. V. Melnikov, "Stackelberg-Nash equilibrium in the linear city model," Automation and Remote Control, vol. 81, no. 2, pp. 358-365, 2020.

[22] Y. T. Zhan, X. S. Li, and N. J. Huang, "A Stackelbergpopulation competition model via variational inequalities and fixed points," Carpathian Journal of Mathematics, vol. 36, no. 2, pp. 331-339, 2020.

[23] S. Sharma, V. Sharma, and A. Goyal, "QoS analysis of multi priority DCF algorithm in wireless local area network," International Journal of Computer Sciences and Engineering, vol. 7, no. 6, pp. 291-294, 2019.

[24] S. Tang, J. Yuan, Z. Zhang, and D. Z. Du, "iGreen: green scheduling for peak demand minimization," Journal of Global Optimization, vol. 69, no. 1, pp. 45-67, 2017. 